\title{
Bioinformatics identification of hub genes and signaling pathways regulated by intravenous immunoglobulin treatment in acute Kawasaki disease
}

\author{
HONGBIAO HUANG*, LEI XU*, YUEYUE DING, JIE QIN, CHENGCHENG HUANG, \\ XUAN LI, YUNJIA TANG, GUANGHUI QIAN and HAITAO LV \\ Institute of Pediatric Research, Children's Hospital of Soochow University, Suzhou, Jiangsu 215025, P.R. China
}

Received October 23, 2020; Accepted March 11, 2021

DOI: $10.3892 / \mathrm{etm} .2021 .10216$

\begin{abstract}
Kawasaki disease (KD) is an acute, self-limiting form of vasculitis commonly encountered in infants and young children. Intravenous immunoglobulin (IVIG) is the primary drug used for the treatment of KD, which may significantly reduce the occurrence of coronary artery lesions. However, the specific molecular profile changes of KD caused by IVIG treatment have remained elusive and require further research. The present study was designed to identify key genes, pathways and immune cells affected by IVIG treatment using multiple bioinformatics analysis methods. The results suggested that myeloid cells and neutrophils were affected by IVIG treatment. Kyoto Encyclopedia of Genes and Genomes pathway analysis identified that hematopoietic cell lineages and osteoclast differentiation may have an important role in the mechanism of action of IVIG treatment. Immune cell analysis indicated that the levels of monocytes, M1 macrophages, neutrophils and platelets were markedly changed in patients with KD after vs. prior to IVIG treatment. The key upregulated genes, including ZW10 interacting kinetochore protein, GINS complex subunit 1 and microRNA-30b-3p in whole blood cells of patients with KD following treatment with IVIG indicated that these IVIG-targeted molecules may have important roles in KD. In addition, these genes were further examined by literature review and indicated to be involved in cell proliferation, apoptosis and virus-related immune response in patients with KD. Therefore, the present results may provide novel insight into the mechanisms of action of IVIG treatment for KD.
\end{abstract}

Correspondence to: Professor Haitao Lv or Dr Guanghui Qian, Institute of Pediatric Research, Children's Hospital of Soochow University, 92 Zhongnan Street, Suzhou, Jiangsu 215025, P.R. China E-mail: 1vhaitao@suda.edu.cn

E-mail: ghqian@suda.edu.cn

${ }^{*}$ Contributed equally

Key words: Kawasaki disease, mechanism of action, intravenous immunoglobulin, bioinformatics analysis

\section{Introduction}

Kawasaki disease (KD) is an acute, self-limiting form of vasculitis that affects infants and children, particularly those aged $<5$ years. In developed countries, KD is the most common cause of acquired heart disease in children (1). Up to $25 \%$ of patients develop coronary artery aneurysm (CAA) if treatment is not provided in a timely manner (2). Since 1983, Furusho et al have used intravenous immunoglobulin (IVIG) to treat patients with $\mathrm{KD}$ in the initial acute phase and the incidence of CAA has decreased from 20-25 to 3-5\% (3-5). However, the mechanisms of action of IVIG treatment for KD have remained elusive. There are several possible mechanisms, including inhibition of inflammatory cytokines, blockade of Fc receptor on macrophages, activation of augmenting T-cell suppressor, inhibition of antibody formation and neutralization of bacterial superantigens (6). Other mechanisms, such as reduced platelet adhesion, decreased oxidative stress and neutrophil apoptosis via a lectin-binding pathway have also been reported (7-9). In an animal model of KD, prevention of T-cell activation and reduction of TNF- $\alpha$ production have been reported (10). In addition, adverse effects have been revealed in the clinical application of IVIG, the most frequent of which include flushing, fever, headache, chills and hemolysis $(11,12)$. In addition, not every family one of patients who suffer from KD in poor countries can afford the expense of treatment. Therefore, the aim of the present study was to investigate the mechanisms of action of IVIG therapy for KD from a genetic perspective. At present, there is a lack of research on genetic changes in patients with KD prior to and after IVIG treatment.

Currently, gene expression profiling microarrays and proteome analysis are widely used to analyze the differential gene expression profiles and enriched pathways in KD. A large amount of gene chip expression profiling microarray data have been published in public databases, such as the Gene Expression Omnibus (GEO) database. In the present study, it was hypothesized that certain genes are associated with IVIG treatment in patients with KD. To test the hypothesis, bioinformatics methods were used to screen out the differentially expressed genes (DEGs) in patients with KD after vs. prior to IVIG therapy. The gene chip data were 
downloaded from the GEO website and the $\mathrm{R}$ project was used to analyze DEGs. Proteome analysis was used to assess the differentially expressed proteins (DEPs). The DEGs and DEPs were combined for Gene Ontology (GO) functional and Kyoto Encyclopedia of Genes and Genomes (KEGG) pathway enrichment analyses. The profiling changes in immune cells were analyzed using xCell toll. A protein-protein interaction (PPI) network was used to determine hub genes from DEGs and the mRNA-microRNA (miRNA/miR) interaction network was established using miRwalk. To further verify the aforementioned results, reverse transcription-quantitative PCR (RT-qPCR) was performed. Potential hub genes and their enriched pathways in patients with KD following IVIG treatment were identified, which provided insight into the mechanisms of action of IVIG in the treatment of KD.

\section{Materials and methods}

Patient data. A total of 50 patients with KD who received IVIG therapy between January 2020 and June 2020 were included in the present study. The whole blood cell samples from the 4 patients with KD were sent for proteomic analysis, which was performed by PTM Biolabs, Inc. They were randomly divided into two groups and the mRNA levels detected in one group and miRNA levels in the other. No significant differences in patient characteristics, including sex, age and duration of fever, were identified between the two groups. The characteristics of the cohort are provided in Table I. Blood samples were collected prior to and $36 \mathrm{~h}$ after IVIG. The study was performed in accordance with the Declaration of Helsinki and approved by the Ethics Committee of the Children's Hospital of Soochow University Suzhou (Suzhou, China; approval no. 2020CS075). All participants and their parents were informed of the study details by the Ethics Committee and provided written informed consent.

Microarray data. In order to identify the DEGs in the post-IVIG KD samples, as compared with the pre-IVIG samples, the GES48498 gene expression profile was retrieved from the National Center for Biotechnology Information (NCBI) GEO database (https://www.ncbi. nlm.nih.gov/geo/). The GES48498 microarray dataset was contributed by Ogihara et al (13), which included 6 whole blood cell samples from patients that had or had not undergone IVIG administration. Expression profiling arrays were generated using the GPL570 Affymetrix Human Genome U133 Plus 2.0 Array (Affymetrix, Inc.).

Identification of DEGs. The raw microarray dataset GES48498 in CEL format was downloaded from the GEO database. The data were pre-processed into expression values using the package Affy (version 1.64.0) of the R project (14). Next, the data were normalized to verify their quality. To screen out the DEGs, the two groups of samples (pre-IVIG and post-IVIG) were analyzed using the Limma package (version 3.42.2) in $\mathrm{R}$ (15). The fold-changes in the expression profiling genes were calculated at the same time. $\mid$ Log fold-changel $>1$ and adjusted $\mathrm{P}<0.01$ were set as the cut-off criteria for the identification of DEGs. All significant DEGs were demonstrated in a volcano plot generated using $\mathrm{R}$.
Proteome analysis. In addition to microarray data, to further understand the mechanism of IVIG in KD, proteome analysis was performed on patients prior to and after IVIG treatment. The American Heart Association defined fever for $36 \mathrm{~h}$ after the first IVIG infusion as IVIG resistance (16). Therefore, the whole blood cell samples from 2 patients with KD were collected prior to IVIG treatment and those of 2 patients with KD were collected $36 \mathrm{~h}$ after IVIG treatment and sent to PTM Biolabs, Inc. for high-throughput quantitative proteome analysis. There was no change in the aspirin dose in any of the patients' blood samples and no glucocorticoids or antibiotics were used. Samples were sonicated three times on ice using a high-intensity ultrasonic processor (Scientz) in lysis buffer (8 M urea, $1 \%$ protease inhibitor cocktail). For PTM experiments, inhibitors were also added to the lysis buffer, e.g. $3 \mu \mathrm{M}$ trichostatin A and $50 \mathrm{mM}$ nicotinamide for acetylation. The remaining debris was removed by centrifugation at $12,000 \mathrm{x} \mathrm{g}$ at $4^{\circ} \mathrm{C}$ for $10 \mathrm{~min}$.The protein sample was then diluted by adding $100 \mathrm{mM}$ tetraethylammonium bromide (TEAB) to achieve an urea concentration of $<2 \mathrm{M}$. Finally, trypsin (Promega Corp.) was added at a trypsin-to-protein mass ratio of 1:50 for the first digestion at $37^{\circ} \mathrm{C}$ overnight and 1:100 trypsin-to-protein mass ratio for a second 4-h digestion at room temperature. After trypsin digestion, the peptide was desalted using a Strata X C18 SPE column (Phenomenex) and vacuum-dried. The peptide was reconstituted in 0.5 M TEAB and processed according to the manufacturer's protocol for the Tandem Mass Tag (TMT) kit (Thermo Fisher Scientific, Inc.). In brief, one unit of TMT/iTRAQ reagent was thawed and reconstituted in acetonitrile. The peptide mixtures were then incubated for $2 \mathrm{~h}$ at room temperature and pooled, desalted and dried by vacuum centrifugation. The tryptic peptides were fractionated by high $\mathrm{pH}$ reverse-phase high-performance liquid chromatography using a Thermo Betasil C18 column (5- $\mu \mathrm{m}$ particles, $10 \mathrm{~mm}$ inner diameter, $250 \mathrm{~mm}$ in length) (Thermo Fisher Scientific, Inc.). In brief, peptides were first separated with a gradient of 8 to $32 \%$ acetonitrile $(\mathrm{pH} 9.0)$ over $60 \mathrm{~min}$ and collected in 60 fractions. Subsequently, the peptides were combined into 6 fractions and dried by vacuum centrifugation. The tryptic peptides were dissolved in $0.1 \%$ formic acid (solvent A) and directly loaded onto a home-made reversed-phase analytical column $(15-\mathrm{cm}$ length, $75 \mu \mathrm{m}$ inner diameter). The gradient was comprised of an increase from 6 to $23 \%$ solvent B $(0.1 \%$ formic acid in $98 \%$ acetonitrile) over $26 \mathrm{~min}, 23$ to $35 \%$ in $8 \mathrm{~min}$ and climbing to $80 \%$ in $3 \mathrm{~min}$ and then a hold at $80 \%$ for the last $3 \mathrm{~min}$, all at a constant flow rate of $400 \mathrm{nl} / \mathrm{min}$ on an EASY-nLC 1000 ultra-performance liquid chromatography (UPLC) system. The peptides were subjected to nitrogen soluble index source followed by tandem mass spectrometry (MS/MS) using a Q Exactive ${ }^{\mathrm{TM}}$ Plus (Thermo Fisher Scientific, Inc.) coupled online to the UPLC. The electrospray voltage applied was $2.0 \mathrm{kV}$. The mass to charge $(\mathrm{m} / \mathrm{z})$ scan range was 350 to 1,800 for a full scan and intact peptides were detected in the Orbitrap at a resolution of 70,000. Peptides were then selected for MS/MS using the normalized collision energy set to 28 and the fragments were detected in the Orbitrap at a resolution of 17,500. A data-dependent procedure that alternated between one MS scan followed by $20 \mathrm{MS} / \mathrm{MS}$ scans with 15.0-sec dynamic exclusion. Automatic gain control was set 
Table I. Clinical characteristics

\begin{tabular}{|c|c|c|c|}
\hline \multirow[b]{2}{*}{ Characteristic } & \multicolumn{2}{|c|}{ Subjects used for RT-qPCR } & \multirow[b]{2}{*}{ P-value } \\
\hline & mRNA & miRNA & \\
\hline Male sex (n) & $15(25)$ & $18(25)$ & 0.157 \\
\hline Age (months) & $29.95(2.09-57.82)$ & $30.76(6.8-54.7)$ & 0.427 \\
\hline $\begin{array}{l}\text { Duration of } \\
\text { fever (days) }\end{array}$ & $6.8(5.57-8.03)$ & $6.76(5.52-8)$ & 0.843 \\
\hline
\end{tabular}

RT-qPCR, reverse transcription-quantitative PCR; miRNA, microRNA.

at $5 \mathrm{E} 4$. The fixed first mass was set as $100 \mathrm{~m} / \mathrm{z}$. The raw data were processed similar to microarray data using the $\mathrm{R}$ project. $\mid$ Log fold-change (FC) $\mid>0.6$ and adjusted $\mathrm{P}<0.05$ were set as the threshold for the identification of DEPs.

GO and KEGG pathway enrichment analysis. To analyze the DEGs and significant proteins for GO term and KEGG pathway enrichment analyses, the ClueGo package (version 2.56) of Cytoscape software (version 3.8.0; https://cytoscape.org/) was used to process data and the package ppglot2 (version 3.3.0) in $\mathrm{R}$ was utilized to visualize the results. GO enrichment analysis included biological process (BP), cellular component (CC) and molecular function (MF) terms. GO annotation and KEGG pathway enrichment analysis of DEGs and DEPs was performed. The intersection part of the pathway was selected to analyze its enrichment in transcriptomic and proteomic analysis, respectively. The adjusted P-value [false discovery rate $(\mathrm{FDR})<0.05$ ] was considered to indicate a statistically significant difference. The top 10 pathways with the greatest difference in transcriptomics and proteomics were selected and the intersection of the two groups was selected as an important GO and KEGG pathway in the treatment of KD by IVIG.

Immune cell analysis. In order to further understand the changes in immune cells prior to and after IVIG treatment, the webtool xCell was used for cell type enrichment analysis of the GES48498 gene expression profile for 64 immune and stroma cell types (17). Given that the distribution of the present data was not normal, the Wilcoxon signed-rank test was used to calculate the difference between two groups with $\mathrm{P}<0.05$ set as the cutoff value.

PPI network and hub gene identification. In order to extract the hub genes from the DEGs in the post-IVIG KD samples, the Search Tool for the Retrieval of Interacting Genes and proteins (STRING) database (version 11.0) was used to calculate and obtain the PPI network (18). A combined score of $\geq 0.4$ for PPI pairs was considered to indicate a statistically significant difference. Visualization was performed with the MCODE (version 1.6.1) plug-in of Cytoscape software (19). The software parameters were set as default. The genes contained in the gene cluster with the highest scores were considered to be the hub genes (20).
Construction of the mRNA-miRNA interaction network. The hub genes were selected and uploaded to the miRWalk 2.0 database to predict its target miRNAs (http://zmf.umm. uni-heidelberg.de/apps/zmf/mirwalk2/). To increase the accuracy of the results, four databases, including TargetScan, miRanda, miRWalk and RNA22 (integrated in the miRWalk 2.0 database), were used to perform the intersection. These four databases are the default databases used to analyse the mRNA-miRNA interaction network. The mRNA-miRNA interaction network was constructed with Cytoscape software (version 3.8.0). The miRNAs that targeted $>3$ genes were screened out for further verification.

Verification of $m R N A$ and miRNA by $R T-q P C R$. To verify the miRNAs and their target hub genes, blood samples were collected from pediatric patients with KD referred to the Children's Hospital of Soochow University (Suzhou, China). mRNA and miRNA were tested in 25 patients. Clinical samples were collected prior to and after treatment with IVIG. Total RNA was extracted using TRIzol ${ }^{\circledR}$ reagent (Thermo Fisher Scientific Inc.). Subsequently, miRNAs were isolated and purified from total RNA using the miRNeasy Mini kit (Qiagen $\mathrm{GmbH}$ ).

mRNA RT was performed using PrimeScript RT Master mix (Takara Bio, Inc.) at $37^{\circ} \mathrm{C}$ for $15 \mathrm{~min}$ and then at $85^{\circ} \mathrm{C}$ for $10 \mathrm{sec}$. The miRNA RT was performed using the miScript II RT kit (Qiagen $\mathrm{GmbH}$ ) at $37^{\circ} \mathrm{C}$ for $60 \mathrm{~min}$ and at $95^{\circ} \mathrm{C}$ for $5 \mathrm{~min}$. The complementary DNA derived from mRNA and miRNA was diluted four times with RNase-free water. Next, a LightCycler 480 II Real-Time PCR system (Roche Diagnostics) was used for the detection of mRNA and miRNA expression levels. qPCR for mRNA quantification was performed with SYBR Green qPCR Master mix (Bimake.com) at $50^{\circ} \mathrm{C}$ for $2 \mathrm{~min}$, followed by 40 cycles at $95^{\circ} \mathrm{C}$ for $15 \mathrm{sec}$ and $60^{\circ} \mathrm{C}$ for $60 \mathrm{sec}$. PCR amplification of miRNA was conducted using the miScript SYBR Green PCR kit (Qiagen $\mathrm{GmbH})$ at $95^{\circ} \mathrm{C}$ for $15 \mathrm{~min}$, followed by 40 cycles at $94^{\circ} \mathrm{C}$ for $15 \mathrm{sec}, 55^{\circ} \mathrm{C}$ for $30 \mathrm{sec}$ and $70^{\circ} \mathrm{C}$ for $30 \mathrm{sec}$. GAPDH and U6 were used as internal controls for mRNA and miRNA, respectively. The relative expression of different samples was calculated using the $2^{-\Delta \Delta C t}$ method (21). Primer sequences were designed by Sangon Biotech Co., Ltd. and the universal primer was provided by Qiagen $\mathrm{GmbH}$. The primer sequences used are listed in Table II.

Cell culture and treatment. Human coronary artery endothelial cells (HCAECs) were purchased from Beijing Beina Chuanglian Biotechnology Research Institute (cat. no. BNCC338441) and cultured with RPMI-1640 medium (Biosharp Corp.) supplemented with $10 \%$ FBS (Biological Industries) and antibiotics $(100 \mathrm{U} / \mathrm{ml}$ penicillin- $\mathrm{G}$ and $100 \mathrm{mg} / \mathrm{ml}$ streptomycin) at $37^{\circ} \mathrm{C}$ in $5 \% \mathrm{CO}_{2}$ and $95 \%$ humidified air. The HCAECs were seeded in each well of a 12-well culture plate at a density of $5 \times 104$ cells and incubated for $18 \mathrm{~h}$ at $37^{\circ} \mathrm{C}$ in $5 \% \mathrm{CO}_{2}$ and 95\% humidified air. The HCAECs were then subjected to $1 \mu \mathrm{g} / \mathrm{ml}$ lipopolysaccharide (LPS; Merck KGaA) challenge for $3 \mathrm{~h}$ (22). Subsequently, the HCAECs were treated with IVIGs (25 mg/ml) (Tonrol Bio-pharmaceutical Co., Ltd.) and vehicle for $18 \mathrm{~h} \mathrm{(23).} \mathrm{The} \mathrm{endothelial} \mathrm{cells} \mathrm{produce} \mathrm{intercellular}$ adhesion molecule 1 (ICAM1) in response to inflammatory 
Table II. Primers designed for real-time PCR.

\begin{tabular}{lll}
\hline Gene & \multicolumn{1}{c}{ Forward (5' to 3') } & \multicolumn{1}{c}{ Reverse (5' to 3') } \\
\hline GAPDH & GGAGAAAC-CTGCCAAGTATG & TTACTCCTT-GGAGGCCATGTAG \\
RRM2 & AGTG-GAAGGCATTTTCTTTCC & GCAAAATCACAGTG-TAAACCCT \\
TK1 & GTTCTCAGGAAAAA-GCACAGAG & GTCTTTGGCATACTT-GATCACC \\
TOP2A & AAGATTCATTGAAGAC-GCTTCG & GCTG-TAAAATGCCATTTCTTGC \\
GINS1 & AGAGCACTCAGATGG-GAATATG & ATCCTGTG-TAATGTCCAAACCT \\
ZWINT & AATT-GCAGCTAAGGAACAATGG & TTTCTCCATGGCCATTT-GTTTC \\
ICAM-1 & ATGCCCAGA-CATCTGTGTCC & GGGGTCTC-TATGCCCAACAA \\
U6 & CTCGCTTCGGCAGCACA & AACGCTTCACGAATTTGCGT \\
miR-3929 & GGAGGCTGATGTGAGTAGACCACT & \\
miR-30b-3p & CGCTGGGAGGTGGATGTTTACTTC & \\
miR-3689 & GCTGGGAGGTGTGATATTGTGGT & \\
miR-485-5p & AGAGGCTGGCCGTGATGAATTC & \\
Universal reverse of miRNAs & 10X miScript Universal Primer (Qiagen GmbH) & \\
\hline
\end{tabular}

ZWINT, ZW10 interacting kinetochore protein; GINS1, GINS complex subunit 1; miRNA/miR, microRNA; ICAM, intercellular adhesion molecule; TOP2A, topoisomerase II alpha; RRM2, ri-bonucleotide reductase regulatory subunit M2; TK1, thymidine kinase 1.
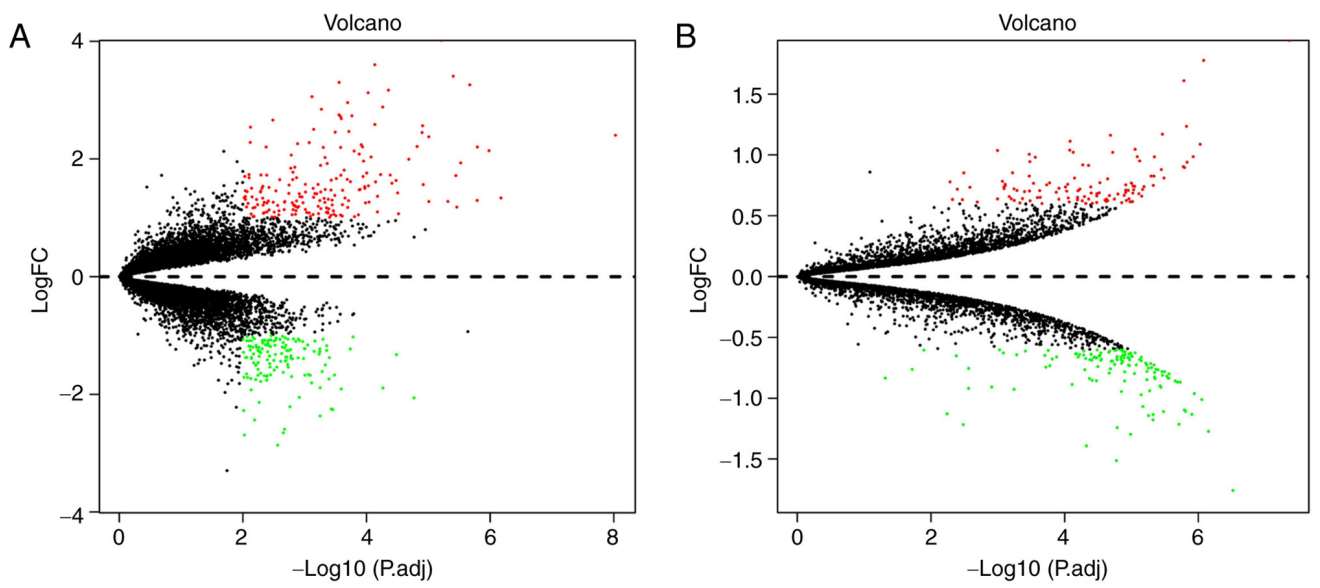

Figure 1. Volcano plot map of (A) differentially expressed genes and (B) differentially expressed proteins. Red dots, upregulated genes/proteins; green dots, downregulated genes/proteins.

cytokines (23). The hub genes in HCAECs were validated by RT-qPCR and imaged using an inverted phase-contrast photo microscope at a magnification of x400 (Olympus IX73; Olympus Corp.). All experiments were repeated at least three times with two replicated each.

Statistical analysis. Data were analyzed using SPSS statistical software for Windows (version 24.0; IBM Corp.) and experimental non-parametric data and parametric data were presented as the mean \pm standard deviation. The Shapiro-Wilk normality test was used to assess the normal distribution of values. The Wilcoxon signed-rank test was used for comparison of categorical data (age and days of fever) and the $\chi^{2}$ test was used for the comparison of categorical data (sex) in Table I $(\mathrm{P}>0.05)$. The differences in mRNA/miRNA expression levels and immune cells between the two groups (pre-IVIG group and post-IVIG group) were analyzed using the Wilcoxon rank-sum test and those between different time-points for the same group with the Wilcoxon signed-rank test, based on the results of the Shapiro-Wilk normality test. For RT-qPCR validation, each group contained 25 samples and each sample was measured by PCR and repeated three times to obtain the mean value. $\mathrm{P}<0.05$ was considered to indicate a statistically significant difference.

\section{Results}

Identification of DEGs and DEPs. To identify DEGs in post-IVIG vs. pre-IVIG KD samples, the raw microarray dataset GES48498 and the annotation of the platform GPL570 were downloaded from the GEO database. DEGs with $\log 2 \mathrm{FCl}>1$ and $\mathrm{P}<0.01$ were determined. A total of $335 \mathrm{DEGs}$ were selected, including 189 upregulated and 146 downregulated genes. According to the results of the proteomic analysis, 253 DEPs were identified, of which 117 were upregulated and 136 downregulated. A volcano plot of the DEGs and DEPs is presented in Fig. 1A and B, respectively. 


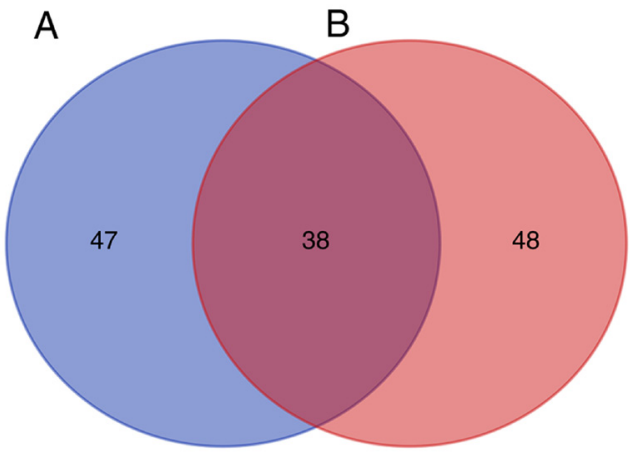

Figure 2. Venn diagrams illustrating the number of GO pathways in DEGs and DEPs. (A) The GO pathways of DEPs are represented by a blue circle. (B) The GO pathways of DEGs are represented by a red circle. The intersection in dark red represents 38 pathways the two groups had in common. GO, gene ontology; DEGs, differentially expressed genes; DEPs, differentially expressed proteins; FC, fold change; P.adj, adjusted P-value.

GO functional and KEGG pathway enrichment analysis. To understand the functions and potential mechanisms of IVIG treatment in KD, the results were analyzed and visualized using Cytoscape software. In total, 335 DEGs and 253 DEPs were included in the analysis. The DEGs were enriched in $86 \mathrm{GO}$ pathways (FDR<0.05), including $68 \mathrm{BP}, 17 \mathrm{CC}$ and $1 \mathrm{MF}$ terms. The DEPs were enriched in $85 \mathrm{GO}$ pathways, including $59 \mathrm{BP}, 20 \mathrm{CC}$ and $6 \mathrm{MF}$ terms. There were 38 pathways at the intersection of the two GO pathway enrichments (Fig. 2). Common GO pathways between DEGs and DEPs were further analyzed. The top 10 genes in each category of the GO pathway enrichment analysis of the DEGs and DEPs according to the FDR are listed in Fig. 3A and B. DEGs enriched in 20 pathways and DEPs enriched in 26 pathways were selected. Next, the intersection of the two groups regarding important pathways was selected and a Venn diagram was drawn to obtain 18 common pathways (Fig. 3C). The DEGs and DEPs were subjected to enrichment analysis of the KEGG pathway and the top 10 pathways are presented in Fig. 4A-C. The results of the GO functional and KEGG pathway enrichment analyses are presented in Tables III-V.

The GO pathway analysis indicated that myeloid cells and neutrophils have important roles in IVIG treatment of $\mathrm{KD}$, including the terms 'granulocyte activation', 'leukocyte degranulation', 'myeloid cell activation involved in the immune response', 'myeloid leukocyte activation', 'myeloid-leukocyte-mediated immunity', 'neutrophil activation', 'neutrophil activation involved in the immune response', 'neutrophil degranulation' and 'neutrophil-mediated immunity'. CC analysis indicated that the DEGs and DEPs were mainly enriched in the terms of 'azurophil granules', 'cytoplasmic vesicle lumina', 'secretory granules', 'secretory granule lumina', 'secretory granule membranes', 'secretory vesicles', 'specific granules', 'specific granule lumina' and 'vesicle lumina'. Furthermore, KEGG pathway analysis demonstrated that DEGs were enriched in 'cytokine-cytokine receptor interaction', 'hematopoietic cell lineage', 'osteoclast differentiation', 'malaria' and 'legionellosis'. The top five KEGG pathways in which DEPs were enriched were 'Type 1 T-helper (Th1) and Th2 cell differentiation', 'Staphylococcus aureus infection', 'hematopoietic cell lineage', 'natural killer cell-mediated
Table III. Important common GO pathways in the enrichment analyses of differentially expressed genes and proteins.

\begin{tabular}{ll} 
A, Category BP & \\
\hline Pathway ID & \multicolumn{1}{c}{ Description } \\
\hline GO:0036230 & $\begin{array}{l}\text { Granulocyte activation } \\
\text { Geukocyte degranulation }\end{array}$ \\
GO:0002275 & $\begin{array}{l}\text { Myeloid cell activation involved in immune } \\
\text { response } \\
\text { Myeloid leukocyte activation }\end{array}$ \\
GO:0002274 & $\begin{array}{l}\text { Myeloid leukocyte mediated immunity } \\
\text { Neutrophil activation } \\
\text { GO:0002444 }\end{array}$ \\
GO:00042119 & $\begin{array}{l}\text { Neutrophil activation involved in immune } \\
\text { response } \\
\text { Neutrophil degranulation } \\
\text { Neutrophil mediated immunity }\end{array}$ \\
GO:0043312 &
\end{tabular}

\section{B, Category CC}

Pathway ID Pathway ID

\begin{tabular}{ll}
\hline GO:0042582 & Azurophil granule \\
GO:0060205 & Cytoplasmic vesicle lumen \\
GO:0030141 & Secretory granule \\
GO:0034774 & Secretory granule lumen \\
GO:0030667 & Secretory granule membrane \\
GO:0099503 & Secretory vesicle \\
GO:0042581 & Specific granule \\
GO:0035580 & Specific granule lumen \\
GO:0031983 & Vesicle lumen \\
\hline
\end{tabular}

GO, Gene Ontology; BP, biological process; CC, cellular component.

Table IV. Kyoto Encyclopedia of Genes and Genomes pathways in the enrichment analyses of dif-ferentially expressed genes.

\begin{tabular}{llrl}
\hline Term & \multicolumn{1}{c}{ Description } & Count & FDR \\
\hline hsa04640 & Hematopoietic cell lineage & 11 & $1.08 \times 10^{-4}$ \\
hsa05144 & Malaria & 7 & $1.62 \times 10^{-3}$ \\
hsa04060 & Cytokine-cytokine receptor & 15 & $1.91 \times 1^{0-2}$ \\
& interaction & & \\
hsa05134 & Legionellosis & 6 & $2.68 \times 10^{-2}$ \\
hsa04380 & Osteoclast differentiation & 9 & $2.72 \times 10^{-2}$ \\
\hline
\end{tabular}

If the analysis results yielded more than five terms enriched in this category, the top five terms were listed according to FDR. FDR, false discovery rate; hsa, Homo sapiens.

cytotoxicity' and 'allograft rejection'. The terms 'hematopoietic cell lineage' and 'osteoclast differentiation' were screened out for both DEGs and DEPs. 
Table V. Top five Kyoto Encyclopedia of Genes and Genomes pathways in the enrichment anal-yses of differentially expressed proteins.

\begin{tabular}{llcr}
\hline Term & \multicolumn{1}{c}{ Description } & Count & FDR \\
\hline hsa04658 & Th1 and Th2 cell differentiation & 11 & $8.03 \times 10^{-6}$ \\
hsa05150 & Staphylococcus aureus infection & 10 & $1.01 \times 10^{-4}$ \\
hsa04640 & Hematopoietic cell lineage & 10 & $1.29 \times 10^{-4}$ \\
hsa04650 & Natural killer cell mediated cytotoxicity & 11 & $2.49 \times 10^{-4}$ \\
hsa05330 & Allograft rejection & 6 & $8.33 \times 10^{-4}$ \\
\hline
\end{tabular}

If the analysis results were more than five terms enriched in this category, the top five terms were listed according to FDR. If the analysis results yielded more than five terms enriched in this category, the top five terms were listed according to FDR. Th1, type 1 T-helper; FDR, false discovery rate; hsa, Homo sapiens.
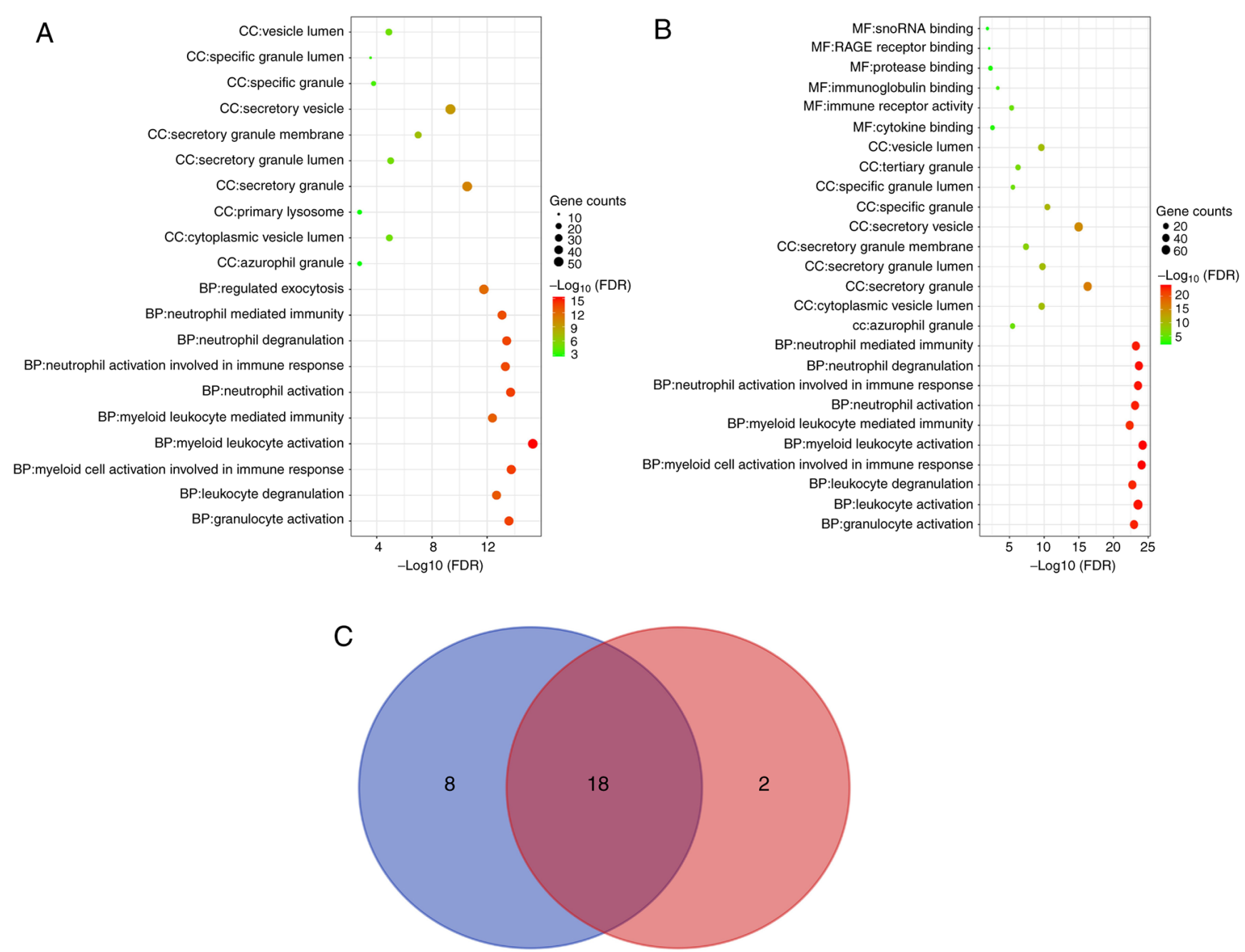

Figure 3. GO enrichment analysis of DEGs and DEPs. For (A) DEGs and (B) DEPs, the top 10 pathways in each GO category (MF, CC and BP) are presented in bubble charts. If the analysis results yielded $>10$ pathways enriched in this category, the top 10 pathways were listed according to the FDR. (C) Venn diagram indicating the GO pathways for the DEGs and DEPs. The top $10 \mathrm{GO}$ pathways for the DEPs in each GO category were determined and represented by a blue circle, while those for the DEGs were represented by a red circle.GO, gene ontology; MF, molecular function; CC, cellular component; BP, biological process; FDR, false discovery rate; DEGs, differentially expressed genes; DEPs, differentially expressed proteins.

Immune cell deconvolution analysis. To obtain a deeper understanding of the potential mechanisms of IVIG treatment for $\mathrm{KD}$, changes in immune cells were analyzed. $\mathrm{xCell}$ was used to perform cell type enrichment based on the gene microarray data. The types of cells that were significantly different following calculation are presented in Fig. 5. A total of eight differential cell types were screened out: Monocytes ( $\mathrm{P}=0.043), \mathrm{M} 1$ macrophages $(\mathrm{P}=0.046)$, neutrophils $(\mathrm{P}=0.043)$, mast cells $(\mathrm{P}=0.043)$, megakaryocyte/erythroid progenitor (MEP) cells $(\mathrm{P}=0.028)$, platelets $(\mathrm{P}=0.028)$, Th1 cells $(\mathrm{P}=0.046)$ and plasma cells $(\mathrm{P}=0.043)$. 

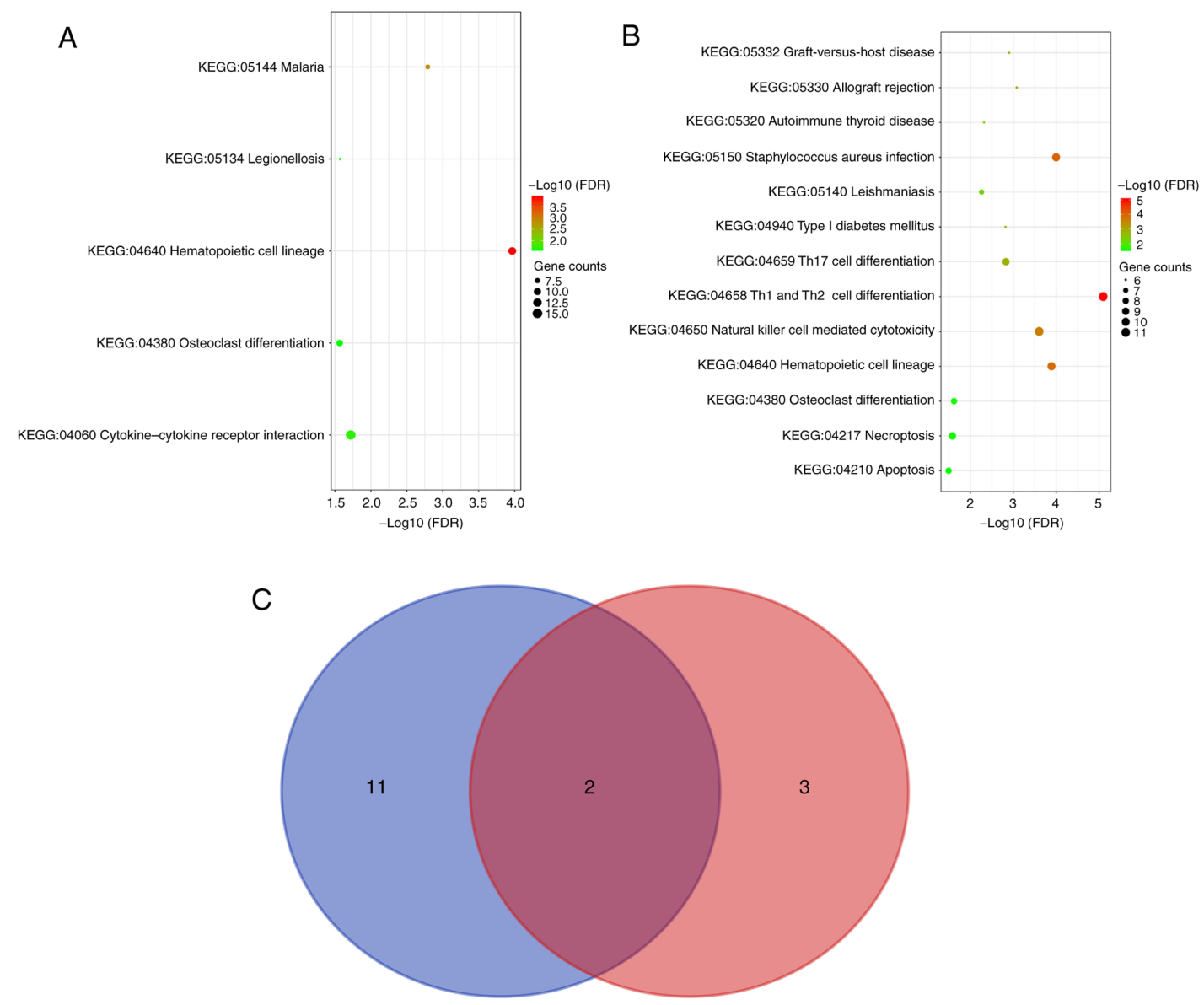

Figure 4. KEGG enrichment analysis of DPGs and DEGs. (A) KEGG enrichment analysis results of (A) DEGs and (B) DPGs presented in bubble charts. (C) Venn diagrams illustrating the number of KEGG pathways for DEGs and DEPs. KEGG pathways calculated for DEPs are indicated by a blue circle and those for DEGs are represented by a red circle. The intersection in dark red represents two pathways that were common between DEGs and DEPs. KEGG, Kyoto Encyclopedia of Genes and Genomes; DEGs, differentially expressed genes; DEGs, differentially expressed genes; DEPs, differentially expressed proteins; FDR, false discovery rate.

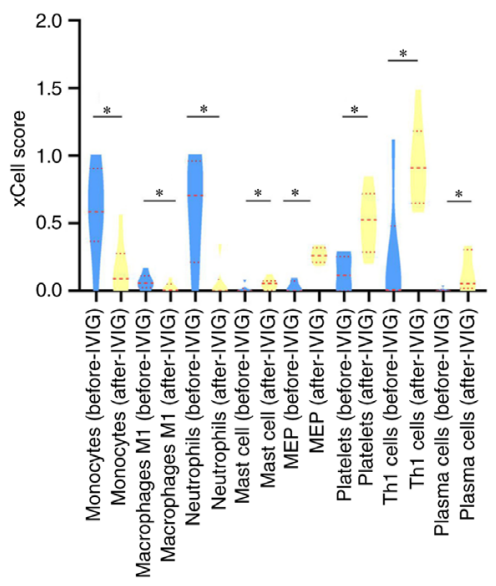

Figure 5. Comparison of the xCell scores of 64 cell types between pre- and post-IVIG treatment whole-blood cell samples of patients with Kawasaki disease in the GES48498 dataset. Violin charts displaying significantly different lymphoid cells. "P<0.05. IVIG, intravenous immunoglobulin; MEP, megakaryocyte/erythroid progenitor cells; Th1, type I T-helper.

PPI network construction and hub gene identification. To screen out the hub genes from a total of 335 DEGs, the online STRING database was used for analysis. The PPI network of the DEGs was visualized using Cytoscape software. The
Table VI. MCODE was used to determine the protein-protein interaction network downloaded from the Search Tool for the Retrieval of Interacting Genes and Proteins database to further mine gene clusters.

\begin{tabular}{lccc}
\hline Cluster no. & Score (density) & Nodes (n) & Edges (n) \\
\hline 1 & 15.75 & 17 & 126 \\
2 & 10 & 10 & 45 \\
3 & 5.684 & 20 & 54 \\
4 & 4.121 & 34 & 68 \\
5 & 3.333 & 4 & 5 \\
6 & 3 & 3 & 3 \\
7 & 3 & 3 & 3 \\
\hline
\end{tabular}

network contained 240 nodes and 932 edges and the average node degree was 6.13. The data were analyzed using Cytoscape software (Fig. 6). The MCODE plug-in of the Cytoscape software was used to calculate the network data to identify gene clusters. The genes in the highest-scoring gene cluster were defined as hub genes. The results of all gene clusters are presented in Table VI. A total of 17 hub genes were screened 


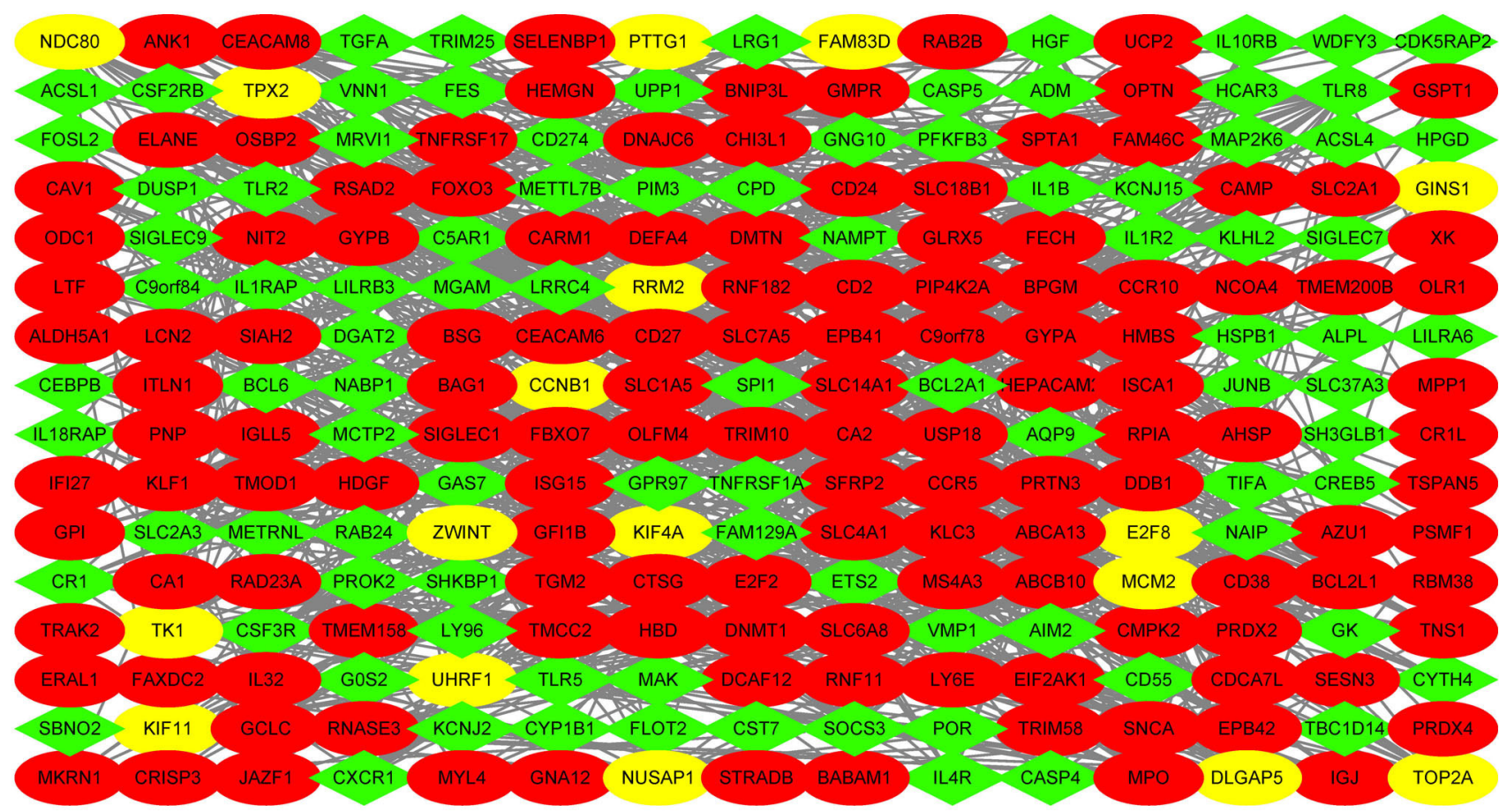

Figure 6. Protein-protein interaction network of differentially expressed genes between the before- and after-intravenous immunoglobulin Kawasaki disease samples. Yellow and elliptical nodes, hub genes; red and elliptical nodes, upregulated genes; green and diamond nodes, downregulated genes.

Table VII. Genes in the highest-scoring gene cluster (Cluster 1) processed by MCODE.

\begin{tabular}{lcc}
\hline Gene/node ID & MCODE score & MCODE cluster \\
\hline ZWINT & 12.752 & Cluster 1 \\
RRM2 & 12.752 & Cluster 1 \\
KIF11 & 12.752 & Cluster 1 \\
KIF4A & 12.752 & Cluster 1 \\
CCNB1 & 12.752 & Cluster 1 \\
E2F8 & 12.752 & Cluster 1 \\
TPX2 & 12.752 & Cluster 1 \\
TOP2A & 12.752 & Cluster 1 \\
NUSAP1 & 12.752 & Cluster 1 \\
MCM2 & 12.752 & Cluster 1 \\
DLGAP5 & 12.752 & Cluster 1 \\
TK1 & 11.868 & Cluster 1 \\
UHRF1 & 11.868 & Cluster 1 \\
NDC80 & 11.868 & Cluster 1 \\
PTTG1 & 11.657 & Cluster 1 \\
GINS1 & 11.000 & Cluster 1 \\
FAM83D & 10.859 & Cluster 1 \\
\hline
\end{tabular}

All of the genes we selected from the highest scoring gene cluster (Cluster 1). ZWINT, ZW10 in-teracting kinetochore protein; GINS1, GINS complex subunit 1; TOP2A, topoisomerase II alpha; RRM2, ribonucleotide reductase regulatory subunit M2; TK1, thymidine kinase 1.

out for further analysis. Those genes are most likely to be essential genes for IVIG treatment of KD. The hub genes are presented in Table VII and Fig. 7.

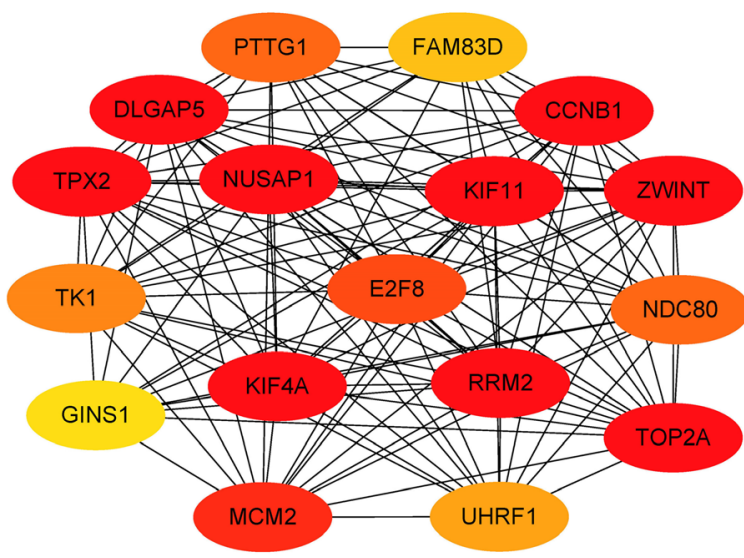

Figure 7. Highest-scoring gene cluster obtained from the protein-protein interaction network with 17 nodes and 126 edges.

Integrated network analysis of mRNA-miRNA interactions. In total, 17 hub genes were uploaded to the online miRWalk 2.0 database to predict the targeted miRNAs. The intersection of miRNA results predicted using the TargetScan, miRanda, miRWalk and RNA22 databases was defined as the prediction result. The parameters were inputted as defaults. The miRNA-mRNA interaction network was constructed and visualized with Cytoscape software. A total of four miRNAs were screened out for further verification, namely miR-3929, miR-30b-3p, miR-3689b-3p and miR-485-5p. The results of the analysis and the interaction network are presented in Table VIII and Fig. 8. All four miRNAs targeted ribonucleotide reductase regulatory subunit M2 and GINS complex subunit 1 (GINS1).

RT-qPCR validation. A total of five mRNAs and four miRNAs were detected and compared in 50 patients with KD. All 




Figure 8. mRNA-miRNA network of hub genes. Yellow and rectangular nodes represent the miRNAs targeting $\geq 3$ mRNAs. Red and elliptical nodes represent mRNAs that interact with miRNAs. Green and diamond nodes represent miRNAs connected to $<3$ mRNAs. miRNA/miR, microRNA.

Table VIII. miRNAs and their target hub genes.

\begin{tabular}{llc}
\hline miRNA & Genes targeted by miRNA & Gene count \\
\hline miR-3929 & RRM2, TK1, TOP2A, GINS1 & 4 \\
miR-30b-3p & ZWINT, RRM2, GINS1 & 3 \\
miR-3689b-3p & ZWINT, RRM2, GINS1 & 3 \\
miR-485-5p & RRM2, TOP2A, GINS1 & 3 \\
\hline
\end{tabular}

ZWINT, ZW10 interacting kinetochore protein; GINS1, GINS complex subunit 1 ; miRNA/miR, microRNA; TOP2A, topoisomerase II alpha; RRM2, ribonucleotidereductase regulatory subunit M2; TK1, thymidine kinase 1 .

blood samples were collected twice, namely prior to and $36 \mathrm{~h}$ after IVIG treatment. A total of 50 (25 for mRNA and 25 for miRNA) samples were tested. Due to the difficulty of collecting blood from children, one sample is not adequate to detect miRNA and mRNA at the same time. The expression levels of ZW10 interacting kinetochore protein (ZWINT), GINS1 and miR-30b-3p differed significantly post- vs. pre-IVIG treatment among the patients with KD. As predicted, the relative expression levels of ZWINT $(\mathrm{P}=0.00446)$ and GINS1 $(\mathrm{P}=0.00029)$ in the pre-IVIG treatment group were lower than those in the post-IVIG treatment group. Both ZWINT and GINS1 were targeted by miR-30b-3p, whose expression level was higher in the pre-IVIG treatment group as compared with that in the post-IVIG treatment group $(\mathrm{P}=0.00012)$ (Fig. 9).

Cell experiment. The HCAECs were stimulated with LPS and underwent IVIG treatment. ZWINT and GINS1 were upregulated, whereas miR-30b-3p was significantly downregulated before and after IVIG treatment in HCAEC. The expression level of ICAM1 was decreased after IVIG treatment. Following LPS stimulation, certain HCAECs exhibited apoptosis, which was assessed by observing the floating cells in the medium. Following IVIG treatment, HCAECs had a faster growth rate as compared with that in the control group (Fig. 10).

\section{Discussion}

$\mathrm{KD}$ is a form of vasculitis that frequently occurs in childhood. IVIG, which may significantly reduce the incidence of CAA in patients with $\mathrm{KD}$, is used as initial therapy (24). In the present study, 335 DEGs and 253 DEPs were identified in patients with $\mathrm{KD}$ after vs. prior to IVIG treatment. Enrichment analysis of 

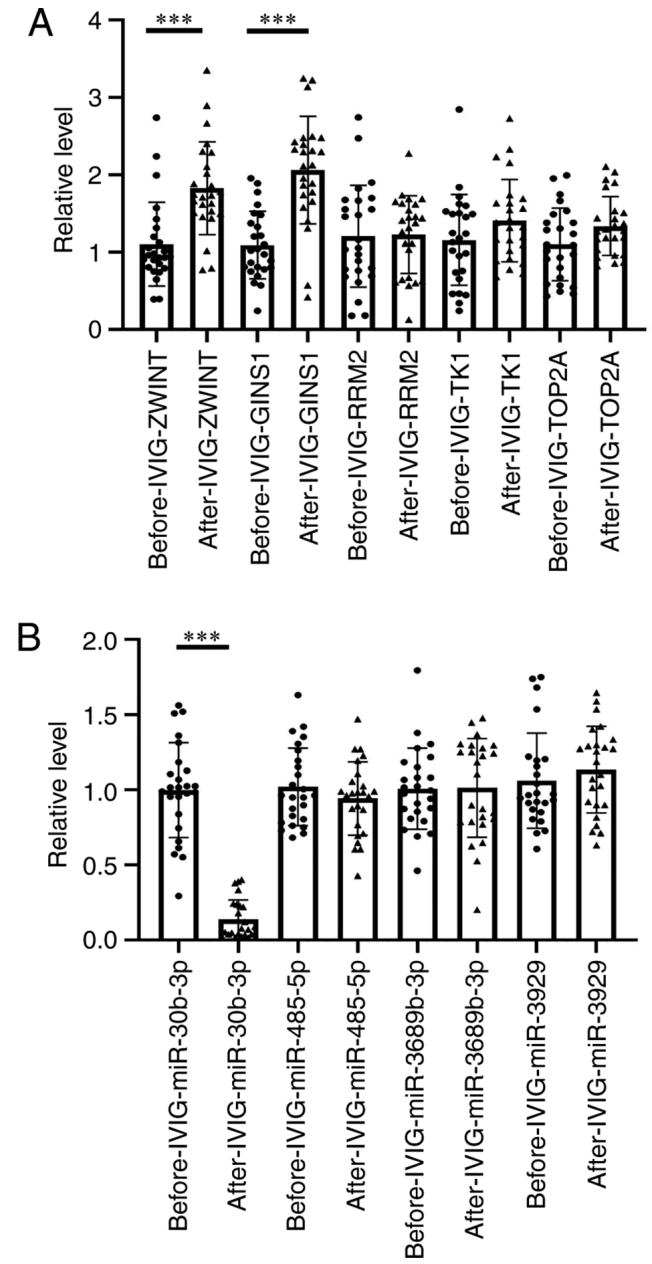

Figure 9. RT-qPCR results. (A) The relative expression levels of ZWINT $(\mathrm{P}=0.00446)$ and GINS1 $(\mathrm{P}=0.00029)$ in a patient with KD prior to IVIG treatment were higher than those after IVIG treatment. (B) The relative levels of miR-30b-3p expression ( $\mathrm{P}=0.00012)$ were lower prior to IVIG treatment compared with those thereafter. ${ }^{* * *} \mathrm{P}<0.01$. Statistical analysis was performed using Wilcoxon's signed-rank test. RT-qPCR, reverse transcription-quantitative PCR; KD, Kawasaki disease; IVIG, intravenous immunoglobulin ZWINT, ZW10 interacting kinetochore protein; GINS1, GINS complex subunit 1; TOP2A, topoisomerase II alpha; RRM2, ribonucleotide reductase regulatory subunit M2; TK1, thymidine kinase 1 .

GO terms and KEGG pathways was performed for the selected DEGs and DEPs. Myeloid cell- and neutrophil-related pathways have been indicated to have an important role in IVIG treatment; these include myeloid cell/neutrophil activation involved in the immune response, myeloid leukocyte/neutrophil activation and myeloid leukocyte/neutrophil-mediated immunity. In addition, through KEGG enrichment pathway analysis, osteoclast differentiation and hematopoietic cell lineage were determined for both DEGs and DEPs, and it was suggested that cell differentiation may have a potential key role in IVIG therapy. Similar to the results of the pathway enrichment analysis, the changes in immune cells were analyzed using xCell tools. A total of eight different types of cells were screened out, including neutrophils and Th1 cells. The PPI and mRNA-miRNA networks were used to screen out hub genes and their target miRNAs of DEGs. Finally, the expression of ZWINT, GINS1 and miR-30b-3p was confirmed to be significantly different between clinical samples and HCAEC
A

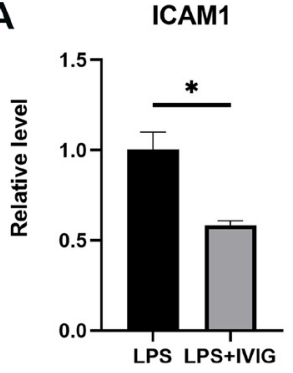

B Hub genes

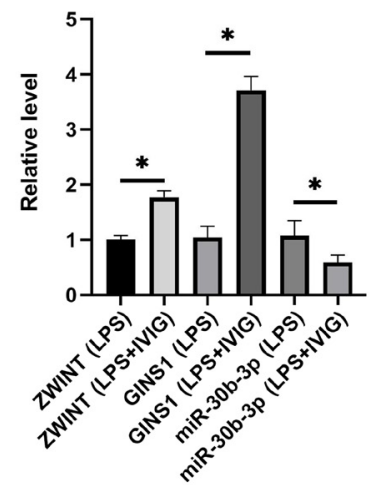

C
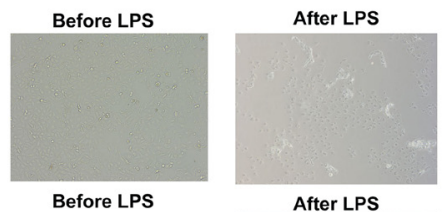

After LPS+1640

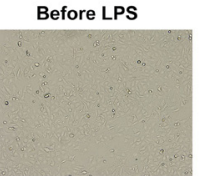

After LPS
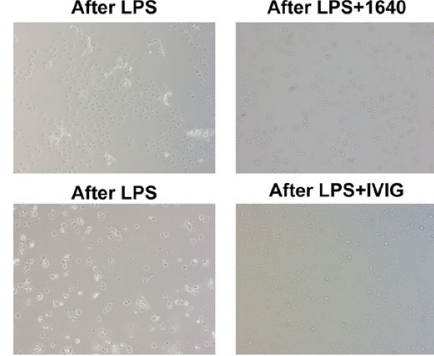

After LPS+IVIG

Figure 10. Results of cell experiment. (A) Relative expression levels of ICAM1 $(\mathrm{P}=0.046)$ in HCAECs stimulated with LPS prior to IVIG treatment were higher than those after IVIG treatment. (B) The relative levels of ZWINT $(\mathrm{P}=0.049)$ and GINS1 $(\mathrm{P}=0.049)$ in HCAECs stimulated with LPS prior to IVIG treatment were lower than those after IVIG treatment. miR-30b-3p expression $(\mathrm{P}=0.043)$ in HCAECs stimulated with LPS prior to IVIG treatment was higher as compared with that after treatment. (C) HCAEC apoptosis was observed following treatment with $1 \mu \mathrm{g} / \mathrm{ml} \mathrm{LPS}$ for $4 \mathrm{~h}$ and cell proliferation was accelerated following treatment with $25 \mathrm{mg} / \mathrm{ml}$ IVIG for $18 \mathrm{~h}$, compared with the control group. Magnification, $\mathrm{x} 400 .{ }^{~} \mathrm{P}<0.05$. Statistical analysis was performed using the Wilcoxon rank-sum test. ICAM1, intercellular adhesion molecule 1; HCAECs, human coronary artery endothelial cells; LPS, lipopolysaccharide; IVIG, intravenous immunoglobulin; ZWINT, ZW10 interacting kinetochore protein; GINS1, GINS complex subunit 1.

by using RT-qPCR analysis. These changes in gene expression were consistent with the predicted results.

The PPI results were analyzed using MCODE in Cytoscape. The gene cluster with the highest score in MCODE was selected. The mRNA-miRNA network and RT-qPCR were used to identify three genes, including two mRNAs and one miRNA. To the best of our knowledge, the association among ZWINT, GINS1, miR-30b-3p and KD has not been previously reported.

ZWINT encodes ZW10 interacting kinetochore protein, which regulates centromere division (25). ZWINT has been reported to regulate the cell proliferation cycle by regulating key mitotic nodes (26). Chromosome instability is also affected by ZWINT (27). Therefore, the present study on ZWINT is mostly focused on tumors, including breast cancer (25), hepatocellular carcinoma (28) and glioblastoma (29). Furthermore, upregulation of ZWINT has a positive regulatory effect on the antiviral signaling pathway (28). Likewise, the relative expression levels of ZWINT were upregulated in the present study. With the upregulation of ZWINT, the proliferation of HCAEC was accelerated. Viral infections such as coronavirus-19 (30) and influenza (31) are considered to be associated with KD. Therefore, whether IVIG regulates the antiviral signaling pathway through ZWINT requires further study. 
GINS1 is also known as Go-Ichi-Ni-San complex subunit 1 or PSF1. DNA replication is closely linked to GINS1 and clinical studies have indicated that GINS1 deficiency may lead to autoimmune disorders, such as neutrophil and NK-cell deficiency (32). However, it has been determined that GINS1 was upregulated and neutrophils were decreased following IVIG treatment. The reason for this may that GINS1 does not directly act on neutrophils. It has been reported that GINS1 may regulate cell proliferation and apoptosis. GINS1 knockdown was indicated to suppress proliferation and accelerate apoptosis in vitro (33), and increased expression of GINS1 may be associated with poor prognosis for patients with cancer, such as breast cancer (34). In the present study, increased expression of GINS1 was indicated to be associated with the proliferation of HCAEC.

miRNAs are short non-coding RNAs associated with cell development, proliferation and apoptosis (35). miR-30b-3p has been reported to be involved in cell inflammation and proliferation through the NF- $\mathrm{B}, \mathrm{T}$-cell receptor (36) and AKT (37) signaling pathways. miR-30b-3p is upregulated in viral diseases, such as respiratory-syncytial-virus-associated pediatric pneumonia (36). With regard to the regulation of tumor cell proliferation, the results vary among different types of tumor. Overexpression of $\mathrm{miR}-30 \mathrm{~b}-3 \mathrm{p}$ promoted apoptosis in ovarian cancer cells (38) and its knockdown suppressed the proliferation of glioma cells (37). The role of miR-30b-3p in prostate cancer remains controversial and the results are also conflicting (39). These differences in miR-30b-3p quantification may be associated with tissue processing, RNA isolation methods, miRNA quantification or miRNA normalization techniques. The present results indicated that these three genes (ZWINT, GINS1 and miR-30b-3p) have a role in regulating the cell proliferation cycle and participating in antiviral immunity. At present, research on IVIG-induced changes in cell proliferation and apoptosis in KD is still lacking. Kato et al (40) reported that immunoglobulin regulated lymphocyte proliferation by suppressing superantigens. According to the present results, ZWINT, GINS1 and miR-30b-3p may be key molecules for the further study of the effect of IVIG in the treatment of KD, since they may affect cell proliferation, apoptosis and virus-related immune response.

Based on the GO analysis, nine BPs and nine CCs were indicated to be significantly different between DEGs and DEPs. Myeloid leukocytes and neutrophils were involved in almost all BPs. The regulation of the activation involved in immune responses of those two cell types may be associated with the mechanisms of action of IVIG in KD. Andreozzi et al (41) indicated that neutrophils were significantly increased in patients with acute-phase KD, since neutrophils in the reserve pool of the bone marrow were released into the peripheral circulation. A 12-year clinical study also confirmed that neutrophils were significantly increased in the acute phase of KD (42). Following IVIG treatment, neutrophils were frequently significantly decreased (42). Data on changes in neutrophils were consistent with these reports $(41,42)$. This indicated that neutrophils may have an important role in the therapeutic mechanisms of IVIG in KD. In addition, several predictive models for IVIG resistance in KD have attempted to identify potential risk factors; the percentage of neutrophils, such as the Kobayashi and Formosa score and certain neutrophil-related ratios, such as the neutrophil-to-lymphocyte ratio, are considered to be associated with IVIG resistance (43). It has also been suggested that the control of neutrophil activation and neutrophil-mediated immune response may be associated with the mechanism of IVIG therapy. Furthermore, the prolonged lifespan of activated neutrophils and prolonged activation of neutrophils may be associated with the pathogenesis of KD and increase the possibility of IVIG resistance $(44,45)$. However, the exact mechanism remains elusive. The results of the GO pathway analysis indicated that hub genes may be involved in regulating the cell proliferation cycle. However, whether IVIG regulates the neutrophil activation cycle requires further study.

Through the KEGG database, the osteoclast differentiation and hematopoietic cell lineage pathway were calculated using ClueGo in Cytoscape. Most of the studies on osteoclast differentiation have focused on rheumatoid arthritis and osteoporosis. Certain transcription factors, such as Jun dimerization protein 2, are able to regulate both osteoclast and neutrophil differentiation. Neutrophils may be linked to the therapeutic mechanism of IVIG (46). A bioinformatics analysis of other microarray data also indicated that osteoclast differentiation was associated with KD (47). Blood-cell development progresses from hematopoietic stem cells. Several cell types, such as neutrophils, T cells, monocytes and macrophages, are derived from hematopoietic cells and are associated with the pathogenesis of KD. The results of immune infiltration analysis of changes in macrophages and monocytes using xCell were consistent with those reported by Sugitani et al (48) and Koizumi et al (49), who confirmed that macrophages and monocytes were upregulated in the acute phase of KD and downregulated following treatment. Similar to macrophages and monocytes, the changing trend in platelets was also consistent with previous studies and the degree of platelet increase was associated with the level of immunoglobulin (50). The role of B cells in the pathogenesis of KD remains elusive. Unlike in the present study, a previous trial reported no changes in the acute and convalescent B-cell subgroups (51). Leung et al (52) reported that the activated Th1 cells were reduced following IVIG treatment in KD. Since only 12 patients were examined and the changing trends in plasma cells came from microarray calculations, the present results may have been accidental and should be confirmed in a large study. A small number of studies on mast and MEP cells in KD are available (53). According to the present analysis of the KEGG pathways and immune cell infiltration, the aforementioned eight types of cells may participate in the mechanism of action of IVIG. Further exploration of the therapeutic mechanisms of IVIG in those cells would be a worthwhile first step for future studies.

The present study had several limitations. First, patients with KD who did not use IVIG were not included as a control group in the study. Due to ethical issues, it is not possible to treat patients with KD without IVIG. Furthermore, the number of samples included in the study was small, in particular the number of samples for proteome analysis. Proteome analysis from 4 different patients was also another limitation of the present study. The results require to be confirmed in a larger study. Finally, certain conclusions came from bioinformatics analyses and require further experimental verification.

In conclusion, in the present study, the mechanisms of action of IVIG in the treatment of KD were analyzed using an integrated bioinformatics analysis. The results indicated that IVIG treatment specifically increased the levels of ZWINT 
and GINS1, and markedly decreased those of miR-30b-3p, and these changes are mainly linked to cell cycle regulation and virus-related immune response. The activation of myeloid leukocytes, neutrophils, monocytes and the M1 type of macrophages, as well as neutrophils and platelets, which are regulated by IVIG, may have an important role in KD. Furthermore, osteoclast differentiation and the hematopoietic cell lineage targeted by IVIG also have a critical role in KD, although these points still require further research.

\section{Acknowledgements}

Not applicable.

\section{Funding}

The present study was supported by grants from the National Natural Science Foundation of China (grant nos. 81971477, 81870365, 82070512 and 81970436), Jiangsu Provincial Medical Young Talents (grant no. QNRC2016756), the Applied Foundational Research of Medical and Health Care of Suzhou City (grant no. SYS2019086), Suzhou Medical Key Discipline Pediatric Cardiology (grant no. Szxk201507), Key Medical Talents in Jiangsu Province (grant no. ZDRCA2016049), Second cycle key subjects of maternal and child health in Jiangsu Province (grant no. FXK201740) and Introduction Project of Clinical Medical Expert Team in Suzhou City (grant no. SZYJTD201805).

\section{Availability of data and materials}

The datasets used and/or analyzed during the current study are available from the corresponding author on reasonable request.

\section{Authors' contributions}

$\mathrm{HH}$ and LX performed the experiments, analyzed the data, prepared figures and/or tables, and authored and wrote drafts of the paper. YD performed the experiments and prepared figures and/or tables. JQ and $\mathrm{CH}$ performed the experiments. $\mathrm{XL}$ and YT prepared figures and/or tables. XL performed the experiments like qRT-PCR, analyzed the data with the software of SPSS. YT prepared figures with Photoshop and helped design and implement cell experiments. GQ and HL conceived and designed the project and revised the final draft. GQ and HL confirm the authenticity of all the raw data. All authors read and approved the final version of the manuscript.

\section{Ethics approval and consent to participate}

This study was approved by the Ethics Committee of the Children's Hospital of Soochow University Suzhou (Suzhou, China; approval no. 2020CS075). Written informed consent was obtained from the guardians of each participant.

\section{Patient consent for publication}

Written informed consent for publication was obtained from the guardians of each participant.

\section{Competing interests}

The authors declare that they have no competing interests.

\section{References}

1. de Graeff N, Groot N, Ozen S, Eleftheriou D, Avcin T, Bader-Meunier B, Dolezalova P, Feldman BM, Kone-Paut I, Lahdenne $\mathrm{P}$, et al: European consensus-based recommendations for the diagnosis and treatment of Kawasaki disease - the SHARE initiative. Rheumatology (Oxford) 58: 672-682, 2019.

2. Lo MS and Newburger JW: Role of intravenous immunoglobulin in the treatment of Kawasaki disease. Int J Rheum Dis 21: 64-69, 2018.

3. Furusho K, Kamiya T, Nakano H, Kiyosawa N, Shinomiya K, Hayashidera T, Tamura T, Hirose O, Manabe Y, Yokoyama T, et al: High-dose intravenous gammaglobulin for Kawasaki disease. Lancet 2: 1055-1058, 1984.

4. Newburger JW, Takahashi M, Beiser AS, Burns JC, Bastian J, Chung KJ, Colan SD, Duffy CE, Fulton DR, Glode MP, et al: A single intravenous infusion of gamma globulin as compared with four infusions in the treatment of acute Kawasaki syndrome. N Engl J Med 324: 1633-1639, 1991.

5. Sosa T, Brower L and Divanovic A: Diagnosis and Management of Kawasaki Disease. JAMA Pediatr 173: 278-279, 2019.

6. Rodriguez MM and Wagner-Weiner L: Intravenous Immunoglobulin in Pediatric Rheumatology: When to Use It and What Is the Evidence. Pediatr Ann 46: e19-e24, 2017.

7. Inagaki $\mathrm{M}$ and Yamada K: Inhibitory effects of high doses of intravenous $\gamma$-globulin on platelet interaction with the vessel wall in Kawasaki disease. Acta Paediatr Jpn 33: 791-798, 1991.

8. Kaneko K, Takahashi M, Yoshimura K, Kitao T, Yamanouchi S, Kimata T and Tsuji S: Intravenous immunoglobulin counteracts oxidative stress in Kawasaki disease. Pediatr Cardiol 33: 1086-1088, 2012.

9. von Gunten S, Schaub A, Vogel M, Stadler BM, Miescher S and Simon H-U: Immunologic and functional evidence for anti-Siglec-9 autoantibodies in intravenous immunoglobulin preparations. Blood 108: 4255-4259, 2006.

10. Lau AC, Duong TT, Ito S and Yeung RS: Intravenous immunoglobulin and salicylate differentially modulate pathogenic processes leading to vascular damage in a model of Kawasaki disease. Arthritis Rheum 60: 2131-2141, 2009.

11. Guo Y, Tian X, Wang X and Xiao Z: Adverse Effects of Immunoglobulin Therapy. Front Immunol 9: 1299, 2018.

12. Zhang G, Xu S, Zhang Z, Zhang Y, Wu Y, An J, Lin J, Yuan Z, Shen L and Si T: Identification of Key Genes and the Pathophysiology Associated With Major Depressive Disorder Patients Based on Integrated Bioinformatics Analysis. Front Psychiatry 11: 192, 2020.

13. Ogihara Y, Ogata S, Nomoto K, Ebato T, Sato K, Kokubo K, Kobayashi $\mathrm{H}$ and Ishii $\mathrm{M}$ : Transcriptional regulation by infliximab therapy in Kawasaki disease patients with immunoglobulin resistance. Pediatr Res 76: 287-293, 2014.

14. Tusher VG, Tibshirani R and Chu G: Significance analysis of microarrays applied to the ionizing radiation response. Proc Natl Acad Sci USA 98: 5116-5121, 2001.

15. Smyth GK: Linear models and empirical bayes methods for assessing differential expression in microarray experiments. Stat Appl Genet Mol Biol 3: e3, 2004.

16. McCrindle BW, Rowley AH, Newburger JW, Burns JC, Bolger AF, Gewitz M, Baker AL, Jackson MA, Takahashi M, Shah PB, et al; American Heart Association Rheumatic Fever, Endocarditis, and Kawasaki Disease Committee of the Council on Cardiovascular Disease in the Young; Council on Cardiovascular and Stroke Nursing; Council on Cardiovascular Surgery and Anesthesia; and Council on Epidemiology and Prevention: Diagnosis, treatment, and long-term management of Kawasaki disease: A scientific statement for health professionals from the American Heart Association. Circulation 135: e927-e999, 2017.

17. Aran D, Hu Z and Butte AJ: xCell: Digitally portraying the tissue cellular heterogeneity landscape. Genome Biol 18: 220-220, 2017.

18. Szklarczyk D, Morris JH, Cook H, Kuhn M, Wyder S, Simonovic M, Santos A, Doncheva NT, Roth A, Bork P, et al: The STRING database in 2017: Quality-controlled protein-protein association networks, made broadly accessible. Nucleic Acids Res 45D: D362-D368, 2017. 
19. Bader GD and Hogue CW: An automated method for finding molecular complexes in large protein interaction networks. BMC Bioinformatics 4: 2, 2003.

20. Yi XH, Zhang B, Fu YR and Yi ZJ: STAT1 and its related molecules as potential biomarkers in Mycobacterium tuberculosis infection. J Cell Mol Med 24: 2866-2878, 2020.

21. Livak KJ and Schmittgen TD: Analysis of relative gene expression data using real-time quantitative PCR and the $2^{-\Delta \Delta C T}$ method. Methods 25: 402-408, 2001.

22. Uthman L, Kuschma M, Römer G, Boomsma M, Kessler J, Hermanides J, Hollmann MW, Preckel B, Zuurbier CJ and Weber NC: Novel Anti-inflammatory Effects of Canagliflozin Involving Hexokinase II inLipopolysaccharide-Stimulated Human Coronary Artery Endothelial Cells. Cardiovasc Drugs Ther: Oct 13, 2020 (Epub ahead of print). doi: 10.1007/s10557-020-07083-w.

23. Armaroli G, Verweyen E, Pretzer C, Kessel K, Hirono K, Ichida F, Okabe M,CabralDA,Foell D,Brown KL, et al: Monocyte-Derived Interleukin-1 $\beta$ As the Driver of S100A12-Induced Sterile Inflammatory Activation of Human Coronary Artery Endothelia Cells: Implications for the Pathogenesis of Kawasaki Disease. Arthritis Rheumatol 71: 792-804, 2019.

24. Chen S, Dong Y, Kiuchi MG, Wang J, Li R, Ling Z, Zhou T, Wang Z, Martinek M, Pürerfellner $\mathrm{H}$, et al: Coronary artery complication in Kawasaki disease and the importance of early intervention: A systematic review and meta-analysis. JAMA Pediatr 170: 1156-1163, 2016.

25. Shao M-T, Hu Y-Z, Ding H, Wu Q, Pan J-H, Zhao X-X and Pan Y-L: The overexpression of ZWINT in integrated bioinformatics analysis forecasts poor prognosis in breast cancer. Trans Cancer Res 9: 187-193, 2020.

26. Dou Z, Prifti DK, Gui P, Liu X, Elowe S and Yao X: Recent progress on the localization of the spindle assembly checkpoint machinery to kinetochores. Cells 8: 278, 2019.

27. Vargas-Rondón N, Villegas VE and Rondón-Lagos M: The role of chromosomal instability in cancer and therapeutic responses. Cancers (Basel) 10: 4, 2017.

28. Zhu Z, Huang S, Zhang Y, Sun C, Tang Y, Zhao Q, Zhou Q, Ju W and $\mathrm{He} \mathrm{X}$ : Bioinformatics analysis on multiple Gene Expression Omnibus datasets of the hepatitis B virus infection and its response to the interferon-alpha therapy. BMC Infect Dis 20: 84, 2020.

29. Yang L, Han N, Zhang X, Zhou Y, Chen R and Zhang M: ZWINT: A potential therapeutic biomarker in patients with glioblastoma correlates with cell proliferation and invasion. Oncol Rep 43: $1831-1844,2020$.

30. Jones VG, Mills M, Suarez D, Hogan CA, Yeh D, Segal JB, Nguyen EL, Barsh GR, Maskatia S and Mathew R: COVID-19 and Kawasaki disease: Novel virus and novel case. Hosp Pediatr 10: 537-540, 2020.

31. Rowley AH and Shulman ST: The epidemiology and pathogenesis of Kawasaki disease. Front Pediatr 6: 374, 2018.

32. Cottineau J, Kottemann MC, Lach FP, Kang YH, Vély F, Deenick EK, Lazarov T, Gineau L, Wang Y, Farina A, et al: Inherited GINS1 deficiency underlies growth retardation along with neutropenia and NK cell deficiency. J Clin Invest 127: 1991-2006, 2017

33. Tang L, Yu W, Wang Y, Li H and Shen Z: Anlotinib inhibits synovial sarcoma by targeting GINS1: A novel downstream target oncogene in progression of synovial sarcoma. Clin Trans Oncol 21: 1624-1633, 2019

34. Fu Y, Zhou QZ, Zhang XL, Wang ZZ and Wang P: Identification of Hub Genes Using Co-Expression Network Analysis in Breast Cancer as a Tool to Predict Different Stages. Med Sci Monit 25 8873-8890, 2019.

35. Ambros V: The functions of animal microRNAs. Nature 431: 350-355, 2004

36. Zhang X, Huang F, Yang D, Peng T and Lu G: Identification of miRNA-mRNA Crosstalk in Respiratory Syncytial Virus-(RSV-) Associated Pediatric Pneumonia through Integrated miRNAome and Transcriptome Analysis. Mediators Inflamm 2020: 8919534 2020 .
37. Jian $\mathrm{Y}, \mathrm{Xu} \mathrm{CH}, \mathrm{Li}$ YP, Tang $\mathrm{B}$, Xie $\mathrm{SH}$ and Zeng EM: Down-regulated microRNA-30b-3p inhibits proliferation, invasion and migration of glioma cells via inactivation of the AKT signaling pathway by up-regulating RECK. Biosci Rep 39: BSR20182226, 2019.

38. Li Y, Zhou J, Wang J, Chen X, Zhu Y and Chen Y: miR-30b-3p affects the migration and invasion function of ovarian cancer cells by targeting the CTHRC1 gene. Biol Res 53: 10, 2020.

39. Kumar B, Khaleghzadegan S, Mears B, Hatano K, Kudrolli TA, Chowdhury WH, Yeater DB, Ewing CM, Luo J, Isaacs WB, et al: Identification of miR-30b-3p and miR-30d-5p as direct regulators of androgen receptor signaling in prostate cancer by complementary functional microRNA library screening. Oncotarget 7 : 72593-72607, 2016.

40. Kato K, Sakamoto T and Ito K: Gamma-globulin inhibits superantigen-induced lymphocyte proliferation and cytokine production. Allergol Int 56: 439-444, 2007.

41. Andreozzi L, Bracci B, D'Errico F and Rigante D: A master role for neutrophils in Kawasaki syndrome. Immunol Lett 184: 112-114, 2017.

42. Sun L, Tang Y, Wang Y, Qian G, Yan W, Wang B, Li X and Lv H: Changes in Profiles of Kawasaki Disease Noted over Time in Suzhou, China. Cardiology 141: 25-31, 2018.

43. Wu G, Yue P, Ma F, Zhang Y, Zheng X and Li Y: Neutrophil-tolymphocyte ratio as a biomarker for predicting the intravenous immunoglobulin-resistant Kawasaki disease. Medicine (Baltimore) 99: e18535, 2020

44. Tsujimoto H, Takeshita S, Nakatani K, Kawamura Y, Tokutomi T and Sekine I: Delayed apoptosis of circulating neutrophils in Kawasaki disease. Clin Exp Immunol 126: 355-364, 2001.

45. Inamo Y, Harada K, Okuni M, Kimoto K, Takeuchi S and Sakurabayashi I: Immunoreactive polymorphonuclear leukocyte elastase in complex with alpha 1-antitrypsin in Kawasaki disease. Acta Paediatr Jpn 29: 202-205, 1987.

46. Maruyama K, Fukasaka M, Vandenbon A, Saitoh T, Kawasaki T, Kondo T, Yokoyama KK, Kidoya H, Takakura N, Standley D, et al: The transcription factor Jdp2 controls bone homeostasis and antibacterial immunity by regulating osteoclast and neutrophil differentiation. Immunity 37: 1024-1036, 2012.

47. Chang D, Qian C, Li H and Feng H: Comprehensive analyses of DNA methylation and gene expression profiles of Kawasaki disease. J Cell Biochem 120: 13001-13011, 2019.

48. Sugitani Y, Furuno K, Sueishi K and Hara T: Macrophages and cytotoxic T cells infiltrate the destructed mitral tissue in Kawasaki disease. BMJ Case Rep: Feb 22 2018: bcr2017223584, 2018.

49. Koizumi K, Hoshiai M, Moriguchi T, Katsumata N, Toda T, Kise H, Hasebe Y, Kono Y, Sunaga Y, Yoshizawa M, et al: Plasma Exchange Downregulates Activated Monocytes and Restores Regulatory T Cells in Kawasaki Disease. Ther Apher Dial 23: 92-98, 2019.

50. Han JW, Oh JH, Rhim JW and Lee KY: Correlation between elevated platelet count and immunoglobulin levels in the early convalescent stage of Kawasaki disease. Medicine (Baltimore) 96: e7583, 2017.

51. Ikeda K, Yamaguchi K, Tanaka T, Mizuno Y, Hijikata A, Ohara O, Takada H, Kusuhara K and Hara T: Unique activation status of peripheral blood mononuclear cells at acute phase of Kawasaki disease. Clin Exp Immunol 160: 246-255, 2010.

52. Leung DY, Burns JC, Newburger JW and Geha RS: Reversal of lymphocyte activation in vivo in the Kawasaki syndrome by intravenous gammaglobulin. J Clin Invest 79: 468-472, 1987.

53. Ross R and Conti P: COVID-19 induced by SARS-CoV-2 causes Kawasaki-like disease in children: Role of pro-inflammatory and anti-inflammatory cytokines. J Biol Regul Homeost Agents 34: 767-773, 2020

(i) $\Theta$ This work is licensed under a Creative Commons Attribution-NonCommercial-NoDerivatives 4.0 International (CC BY-NC-ND 4.0) License. 